\title{
Overview of CPL and LHP Applications on NASA Missions
}

\author{
Dan Butler \\ Thermal Engineering Branch, Code 545, NASA Goddard Space Flight Center, Greenbelt, Md. 20771 \\ Phone: (301) 286-3478 E-mail: Dan.Butler@gsfc.nasa.gov
}

\begin{abstract}
Capillary Pumped Loops (CPL's) and Loop Heat Pipes (LHP's) are finding increased acceptance on upcoming NASA spacecraft missions, as well as military and commercial applications. The transition from a research and development tool to an "off the shelf" system is underway. The state of the art of two phase systems (TPS) is reviewed and applications on various NASA missions are discussed, with particular emphasis on new technology developments. Upcoming research areas and flight experiments are also addressed, along with recommendations for future activities.
\end{abstract}

\section{INTRODUCTION}

Proper communication between Spacecraft project managers and two-phase technology specialists is critical in order to gain acceptance of two-phase systems (TPS) and to insure that the applications are appropriate and implemented correctly. Two-phase specialists are being called on for flight project support as the emphasis (and funding) for technology development has shifted from basic research to near term applications. Nonetheless, parallel efforts are being pursued to continue to advance the state of the art of TPS and maintain a long term research and development program.

\section{TWO PHASE SYSTEMS - STATE OF THE ART}

\section{Starter Pump CPL's}

Single pump CPL's, also known as "Starter Pump CPL's", have been flight proven via the CAPL 2 flight experiment (Ku, 1996). The "Starter Pump" designation refers to the original use of this pump design as a mechanism to start a multiple pump CPL. Later, a CPL was developed based on a single starter pump, which utilizes a direct connection from the reservoir feed line to the core of the pump (Figure 1). This configuration provides a "self-priming" effect at system startup since the liquid must pass through the pump core as it is pushed into the reservoir. A sweeping action is produced that flushes vapor bubbles out of the pump core and promotes successful startups. Starter Pump CPL's have proven to be very reliable and are currently baselined for instrument thermal control on the EOS-AM and HST SM-3 missions, which are described later in this paper.

\section{Multiple Pump CPL's}

Multiple pump CPL's are needed for applications requiring multiple heat acquisition zones and/or heat sharing capability (allows heat removal from the loop). A multiple pump CPL could serve as a central thermal bus on a spacecraft, providing heat removal at several locations as well as providing a heat source for various instruments and electrical components (Figure 2). Flight verification of multiple pump loops is required before these systems can be baselined for spacecraft thermal control. The CAPL 3 flight experiment will fulfill this need by flying a "traditional" CPL system that has four pumps plus a starter pump (Ku, 1998). A back-pressure regulator is used in the vapor line to insure proper startup of the loop in microgravity and provide heat sharing capability. Although 


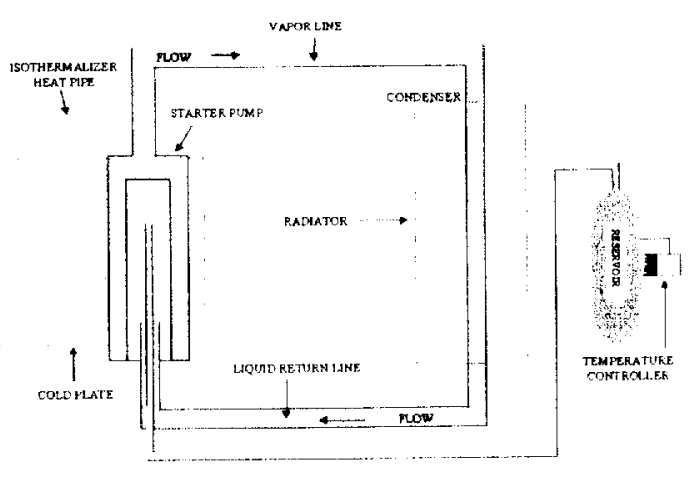

FIGURE 1. Starter Pump CPL

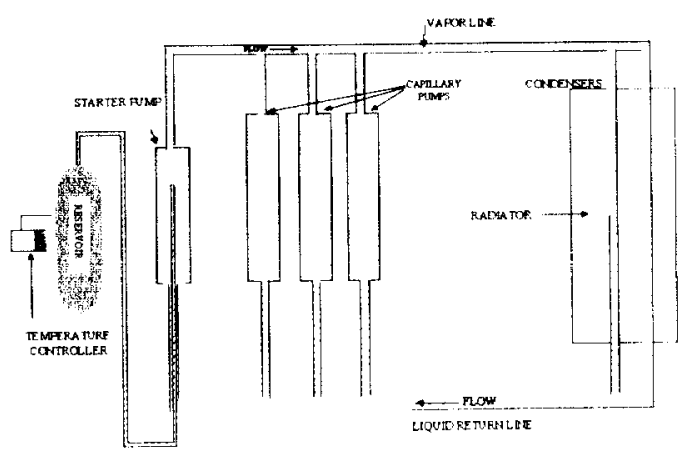

FIGURE 2. Multiple Pump CPL

the TRW Two-Phase Flow (TPF) mission flew multiple pumps, it did not incorporate all of the features that are needed for a typical system, such as a starter pump (Ottenstein, 1998). A reflight of TPF with a starter pump is in work to support a possible multiple pump CPL application on the EOS-Chemistry mission.

\section{Loop Heat Pipes}

The year 1997 was a banner year for Loop Heat Pipes with both Dynatherm and Swales conducting successful flight experiments (Baker, 1998; Yun, 1998). LHP's are now considered flight proven and are baselined for upcoming communications satellite applications and two NASA missions. There are several differences between LHP's and CPL's, even though both have the same general operating principles. LHP's have a single evaporator that is integral with the compensation chamber, as compared to CPL's, which have a separate reservoir and reservoir feed line (Figure 3). LHP's are particularly useful where loop temperature control is not required, thus negating the need to provide separate heater controller electronics. However, they can provide temperature control if needed, as was demonstrated on the Dynatherm LHP mission, although LHP's require more power for this feature than CPL's. For a CPL, the reservoir heater only has to overcome the parasitic heat losses from the reservoir (plus startup transients). For the LHP, the compensation chamber power needs to be sufficient to heat all of the returning fluid to the desired temperature level and overcome subcooling. Another limiting fealure of LHP's is the development of multiple pump loops, which is expected to be much more difficult to accomplish than multiple pump CPL's. LHP's, however, have demonstrated a distinct advantage over CPL's in pumping capability due to the use of metal wicks with smaller pore sizes. LHP's have been built with 1.5 micron wicks that have a 4.5 meter pumping height, while typical CPL's have 15 micron wicks with a 0.45 meter pumping height. Application of metal wick technology to CPL 's has been difficult so far, with the current generation of CPL's limited to plastic 


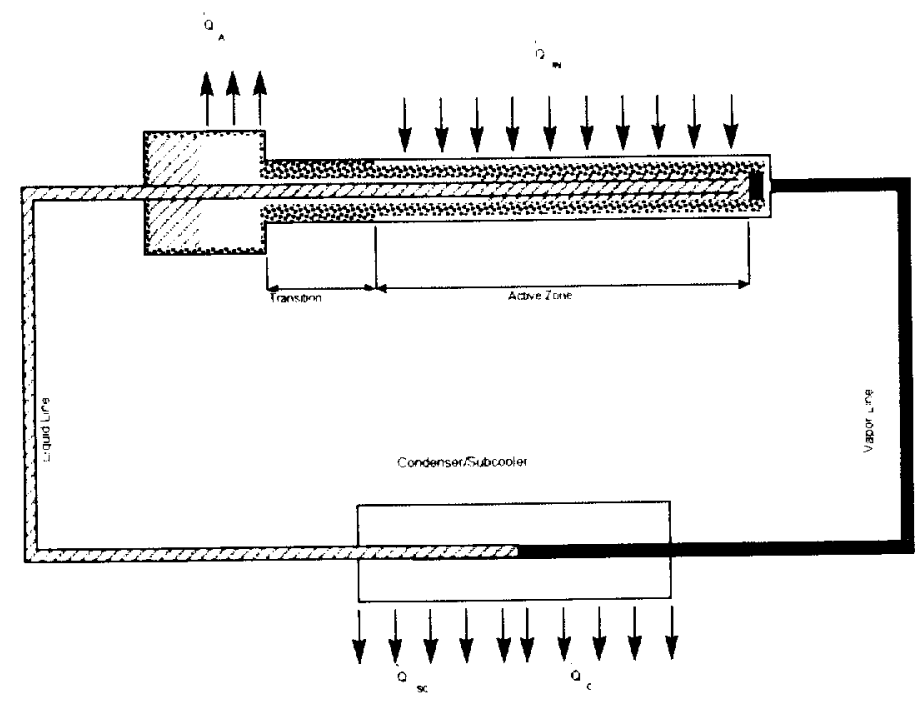

FIGURE 3. Loop Heat Pipe

polyethylene wick material. Both CPL's and LHP's will be used on future missions, depending on individual spacecraft requirements, with each system demonstrating distinct advantages and disadvantages. The project personnel and two-phase technologists must examine each application carefully to select the appropriate technology solution.

\section{APPLICATIONS OF TWO-PHASE SYSTEMS ON NASA MISSIONS}

\section{Earth Observing System (EOS-AM) Spacecraft}

Six starter pump CPL's are being used for the thermal control of 3 instruments (each instrument has a redundant loop) on the EOS-AM Spacecraft (Fredley, 1992). CAPL 2 was the prototype of the EOS-AM CPL system. All CPL testing has been successful so far, including engineering model, flight unit tests, spacecraft thermal vacuum, and the life test unit (which has been running continuously in excess of 2 years). The EOS-AM launch was originally planned for July 98, but has been delayed until 1999.

\section{Earth Observing System Chemistry Mission (EOS-CHEM)}

Due to radiator sizing and power limitations, a multiple pump CPL system built by TRW has been considered for the thermal control of the Microwave Limb Sounder (MLS) instrument on the EOS-CHEM mission, planned for launch in late 2002. Two redundant loops would be employed, each with two pumps plus a starter pump in parallel. The proposed TRW starter pump configuration does not use the same plumbing arrangement employed by the Swales starter pump loop. It utilizes a starter pump plumbed in parallel to the flight pumps, but does not have a direct connection to the reservoir feed line. The Two Phase Flow Flight Experiment (TPF) will be modified and reflown as a flight verification of this system. The new experiment is named the EOS-CHEM CPL Qualification Flight (CCQ) and should be flight ready in mid 1999.

Another potential application of two-phase technology on the EOS-CHEM mission is found on the Tropospheric Emission Spectrometer (TES) instrument. Use of a LHP to carry heat from the instrument to a dedicated radiator is being investigated. This will greatly improve mechanical packaging of the instrument components thus reducing its weight. The LHP will also substantially reduce the survival heater power requirements by thermally decoupling the instrument from the radiator when the instrument is off. 


\section{Geoscience Laser Altimeter System (GLAS)}

The GLAS instrument has baselined a single LHP that interfaces with standard heat pipes to cool 3 lasers. The LHP has a dedicated radiator and interfaces with the heat pipes via a saddle attached to the evaporator. A proportionally controlled heater on the compensation chamber will provide temperature control. The use of a LHP on GLAS substantially reduces the survival heater power that would be needed if a TPS was not used. A prototype of the loop has been successfully tested at GSFC, and has proven the feasibility of the proposed design. GLAS recently completed a preliminary design review, with a flight planned after 2000.

\section{Hubble Space Telescope Servicing Mission 3 (HST SM-3)}

The next HST servicing mission calls for the installation of five starter pump CPL's during astronaut ExtraVehicular Activities (EVA's) in early 2000. This mission has provided a substantial challenge since the loops have to be designed for on-orbit installation of the hardware onto the HST. Four of the loops will provide cooling to HST instruments while a $5^{\text {th }}$ loop will be used to cool a cryocooler to be installed on the NICMOS instrument (McIntosh, 1998). The mission will be preceded by the HST Orbital Systems Test (HOST) shuttle flight experiment in October 98 to verify proper operation of the Cryocooler/CPL hardware in microgravity. Ground testing of the HOST CPL has been successfully completed, and some interesting new features have been demonstrated:

1. The reservoir line is embedded in the liquid line, thus eliminating a separate subcooling requirement for the reservoir line. Also, the plumbing needs are reduced from 3 lines per loop to 2.

2. Loop temperature set point control was demonstrated based on measured liquid line subcooling, rather than reservoir temperature, as has been traditionally done on CPL's.

3. The loop contains 15 meter long liquid and vapor lines, which are primarily constructed out of flex lines rather than traditional hard stainless steel lines. This allows for on-orbit installation and offers substantially increased design and integration flexibility for other future CPL applications.

4. The loop was tested at temperature set points as low as $-10 \mathrm{C}$ to $-20 \mathrm{C}$. It was discovered that significant pump superheat $(>10 \mathrm{C}$ for high power) occurs on a standard pump design below $-10 \mathrm{C}$. This is due to the coefficient of thermal expansion (CTE) mismatch between the polyethylene wick and the aluminum pump body (Porex CTE is approximately 8 times that of aluminum). As the temperature drops, the wick loses contact with the pump body, thus leading to superheat. Decreased wick manufacturing tolerances have reduced this problem at $-10 \mathrm{C}$, but it is still a concern below these temperatures.

\section{MARS-98}

This mission originally utilized two starter pump CPL's for thermal control of the Mars-98 spacecraft batteries and electronics (Clayton, 1997). Several unique features included operation in Mars gravity $(0.38 \mathrm{G})$ at tilt angles up to 16 degrees. Also, a "fixed conductance" mode was to be employed above $-5 \mathrm{C}$ to reduce radiator size requirements, and a deprime heater was to be used to shutdown the loop for cold overnight "sleep periods." However, difficulties arose with the implementation of the Mars98/CPL during Spacecraft Integration and Test, and a passive thermal design has been employed instead. The use of a CPL for this application was not appropriate due to a number of technical reasons. Unfortunately, this was not recognized until late in the spacecraft integration phase due to a lack of communication and the lack of a thorough understanding of CPL requirements. Some of the difficulties with this application include the following:

1. One-g testing included startups at a 16.5 inch adverse elevation. This led to "stressful" startups since the total pumping capability of the wicks was approximately 20 inches, resulting in possible vapor injection into the core of the pump and a pump deprime. 
2. There was no subcooling provided for the reservoir line. This line was located in an environment that was potentially warmer than the loop operating temperature, leading to possible vapor generation in the line and eventual deprime of the pump.

3. Fixed conductance mode at startup - This means that the reservoir is hard filled with liquid during the startup. Previous CPL's have always used a variable conductance mode, which allows for vapor generated in the core of the pump to be flushed into the reservoir line and the reservoir. In the fixed conductance mode, the vapor remains in the pump core, which can lead to a pump deprime. Another issue with the fixed conductance mode is increased sensitivity to Non-Condensible Gas (NCG), especially with the small radiator volumes present in this design.

4. No startup heaters/large thermal mass - Startup heaters that are attached directly to the pump body are typically used to start up a loop, but in this case, no starter heaters were available. Also, a large thermal mass was attached directly to the cold plate. The combination of these factors resulted in heating of the pump very slowly at startup, leading to a loss of subcooled liquid in the pump. It also resulted in a large superheated mass at startup, which increased the "stress level" on the pump, thus increasing the possibility of vapor generation in the core and subsequent pump deprime.

5. Component level testing of the loop did not accurately reflect the conditions that would be encountered in the spacecraft application.

BOTTOM LINE - Although two phase systems have a lot to offer, they still have limitations that must be understood for each potential application. It is imperative that two-phase specialists are included in the decision making processes for applications of two-phase systems and that project personnel are properly educated on their operations and behavior.

\section{UPCOMING FLIGHT EXPERIMENTS}

Two phase systems technology development is continuing with several flight experiments in work. The HOST and CCQ missions are basically prototypes for specific spacecraft applications and have been described above. In addition, two technology development missions are in work, one in the cryogenics area, and the other provides a microgravity demonstration of a multiple pump CPL. They are described below:

\section{Cryogenic Thermal Storage Unit (CRYOTSU)}

The CRYOTSU experiment includes a cryogenic CPL (CCPL) that has nitrogen as the working fluid with operating temperatures in the 75 to $100 \mathrm{~K}$ range (Bugby, 1998). Potential CCPL applications include cooling of cryogenic sensors and detectors, and the experiment includes a breadboard superconducting bolometer. CCPL's have been successfully ground tested with both nitrogen and neon ( 35 to $40 \mathrm{~K}$ ) working fluids. The CRYOTSU testbed also includes a Thermal Storage Unit (TSU) that utilizes the solid/liquid phase change of Nitrogen (at 63 $\mathrm{K}$ ) as a thermal storage device. Thus, CRYOTSU employs both solid/liquid and liquid/vapor nitrogen phase change experiments for future thermal control applications. It is manifested on the STS-95 shuttle mission with a scheduled flight in October 98.

\section{Capillary Pumped Loop (CAPL 3)}

The CAPL 3 experiment is a test of a multiple pump loop of the "traditional" design ( $\mathrm{Ku}, 1998)$. It includes 4 parallel pumps and a starter pump (Swales design) and will reuse the CAPL test bed. New features of CAPL 3 include: (1) A back-pressure regulator located in the vapor line near the condenser. It is used to clear the pump grooves for startup and provide heat sharing capability to an inactive pump. (2) The reservoir heat sink is provided by a liquid cooled shroud. This allows reservoir placement internal to a spacecraft. (3) Fixed conductance mode the ammonia charge inventory is increased compared to traditional levels so that the loop enters a fixed conductance mode above a predetermined reservoir set point temperature. This provides a gradual loss of temperature control (temperature increase) rather than an abrupt deprime if the condenser limit is exceeded. Loop 
startup and normal operation will be done in the traditional variable conductance mode. CAPL 3 is tentatively manifested on a Space Station Shuttle mission in early 2000 although it is expected to be flight ready by the middle of calendar year 99.

\title{
RECOMMENDED FUTURE ACTIVITIES
}

\author{
Room Temperature Loops
}

PUMP DIAMETER - The CAPL 1 and TPF flights both have shown that a reliable CPL with smaller diameter pumps is not yet available. The large diameter pumps (approximately 1 inch) were very reliable on the TPF mission and even started under fully flooded conditions (Ottenstein, 1998). However, the small diameter pumps (approximately $1 / 2$ inch) continue to demonstrate startup difficulty and operational reliability problems. Successful demonstration of reduced size CPL's is needed as future spacecraft shrink in size, yet exhibit increased power densities. Therefore, miniaturized CPL's and LHP's are strong candidates for future NASA spacecraft. The miniaturization effort already undertaken for cryogenic CPL's can be leveraged for room temperature applications. Mini-CPL systems will have to be flight proven before they are implemented, especially due to the known difficulties that have been encountered with small diameter pumps. Miniaturized LHP's are of interest as well with both mini-CPL's and mini-LHP's having potential applications for spacecraft, instrument and electronics cooling. One potential application is the Nanosat program, which is envisioned as a constellation of satellites, with each one weighing less than 10 kilograms and measuring 12 inches in diameter.

WICK MATERIALS - Metal wick pumps have already been demonstrated on LHP's and provide greatly improved pumping height and pressure head compared to polyethylene wicks. Incorporation of metal wick pumps on room temperature CPL's can greatly improve their performance, however, manufacturing concerns and wick thermal conductivity (loss of subcooling) still need to be addressed for CPL applications. Other possibilities include the Sol-Gel wick development work at the Naval Research Lab.

LHP's - Demonstration of multiple pump LHP's and high performance (high power density) LHP's can greatly enhance the potential applications of this technology. There is currently a Phase 2 SBIR underway to build and test high power density LHP's which would have applications in such areas as high powered lasers

ACPL's - The Advanced CPL is designed to be tolerant of vapor bubbles in the core of the pump. A separate evaporator pump located near to or integral with the reservoir provides a "forced priming" of the pump. There is currently a Phase I SBIR underway to promote this effort.

\section{Cryogenic Loops}

Cryogenic CPL's and LHP's can theoretically be developed to work at several different temperature ranges, depending on the working fluid utilized. A substantial effort has already been undertaken for CCPL's, but work on cryogenic LHP's has not reached the same level of maturity. Flight of a neon CCPL can provide microgravity verification of the loop in the 35 to $40 \mathrm{~K}$ range, however, substantial modifications to the existing Cryo-test bed would be required to reach these temperatures. One potential application of a neon loop is the Next Generation Space Telescope (NGST), which may have thermal control requirements that can only be met with a CCPL operating in this temperature range (or lower). Another area of interest for CCPL development is the 180 to $200 \mathrm{~K}$ temperature range, which could be accomplished with a propane working fluid. These levels can be easily reached with the existing test bed and would be a prime candidate for a follow-on Cryogenic test bed mission. Use of Helium to reach temperatures on the order of 2 to $4 \mathrm{~K}$ is also a possibility, although implementation of this loop has obvious technical challenges. A potential application for a helium loop is cooling of a proposed large optical infrared instrument that would be maintained in the $3 \mathrm{~K}$ temperature range. 


\section{SUMMARY}

Applications of two-phase systems are continuing to increase as the technology matures and becomes accepted by the spacecraft community. Order of magnitude performance gains over traditional thermal control techniques is leading to substantial weight reduction and power savings, and more efficient spacecraft designs. Efficiency improvements should improve further as instrument and spacecraft designs utilize a TPS early in the design cycle, rather than as a stop-gap measure after the hardware has been built. Care needs to be taken when employing TPS's as their limitations need to be completely understood and conditions unique to each application have to be examined. For example, sufficient subcooling is required for TPS operation, therefore, knowledge of the expected operating environment is needed before the TPS can be implemented. Design adjustments may be necessary to accommodate the TPS. Future TPS applications will include use in terrestrial areas as well, with TPS currently being studied for thermal control of aircraft de-icing, tank avionics cooling, transformer heat dissipation, and manned applications (space suits, fire suits, etc.).

\section{DISCLAIMER}

The opinions expressed in this document are those of the author and do not necessarily represent an official NASA position.

\section{REFERENCES}

Baker, C., Bienert, W., and A. Ducao, "Loop Heat Pipe Flight Experiment," SAE Paper No. 981580, 1998

Bubgy, D., et. al. , "Development and Testing of a Cryogenic Capillary Pumped Loop Flight Experiment," IECEC Paper No. 98-1288, 1998.

Clayton, S., Martin, D., and J. Bauman, "Mars Surveyor Thermal Management Using a Fixed Conductance Capillary Pumped Loop," SAE Paper No. 972467, 1997.

Fredley, J., and A. Pelszynske, "Accommodation of the EOS-AM Instrument Set Using Capillary Pumped Heat Transport Technology," SAE Paper No. 921404, 1992

Ku, J., Ottenstein, L., and D. Butler, "Performance of the CAPL 2 Flight Experiment," SAF, Paper No. 961432, 1996.

Ku, J., Ottenstein, L., Cheung, K., Hoang, T., Yun, J., "Ground Tests of Capillary Pumped I.oop CAPL 3) Flight Experiment," SAE Paper No. 981812, 1998.

McIntosh, R., Kaylor, M., Buchko, M., and E. Kroliczek, "A Capillary Pump Loop Cooling System for the NICMOS Instrument," SAE Paper No. 981814, 1998

Ottenstein, L. and J. Nienberg, "Flight Testing of the Two-Phase Flow Flight Experiment," SAE Paper No. $981816,1998$.

Yun, J. "American Loop Heat Pipe (ALPIIA) Flight Experiment," CPI 98 International Workshop, 1998. 